\title{
Study of Drag Reduction Due to Aqueous Sodium Polyacrylate by the Rolling Ball Method
}

\author{
Fumitaka YAMASHITA, Kunio NAKAMURA, and Tsurutaro NAKAGAWA \\ Department of Polymer Science, Faculty of Science, \\ Hokkaido University, Nishi 8-chome, Kita 10-jo, \\ Kita-ku, Sapporo 060, Japan
}

(Received December 6, 1982)

\begin{abstract}
A new apparatus was constructed by modifying the rolling ball viscometer in order to study drag reduction due to polymer solutions. Using this apparatus, dilute aqueous solutions of sodium polyacrylate $(200 \mathrm{ppm})$ containing various amounts of $\mathrm{KNO}_{3}$ were examined at $25^{\circ} \mathrm{C}$ in the region of the Reynolds number where drag reduction begins to appear. The drag coefficient and Reynolds number were calculated using the equations proposed by Hubbard and Brown. It was found that drag reduction is markedly affected by the concentration of added $\mathrm{KNO}_{3}$ and that the maximum effect appears at $5.00 \times 10^{-3} \mathrm{moll}^{-1}$ of $\mathrm{KNO}_{3}$. This finding may be favorably explained by Kohn's energy storage theory.

KEY WORDS Dilute Solution Property / Drag Reduction / Rolling Ball Method / Sodium Polyacrylate / Added Salt /
\end{abstract}

Since 1948 when Toms ${ }^{1}$ first observed drag reduction due to polymer solutions, this phenomenon has been investigated by many authors, mainly in regard to pipe flow. However, a large-scale instrument and large volume of sample fluid are needed for this, and besides the difficulty in studying the temperature effect on this phenomenon. In a previous paper, ${ }^{2}$ we constructed a more convinient apparatus by modifying the rolling ball viscometer and showed that the falling velocity of the ball is larger in a polymer solution than in its solvent, pure water. In the present paper, we report the results from a drag reduction study performed with the same apparatus in the region around the critical Reynolds number. Very few studies have been carried out in this region.

There are two types of falling ball viscometers. In one, the ball simply translates vertically in a sample fluid, and in the other, the ball falls, rolling on the wall of an inclined pipe filled with a sample fluid. Initially, we constructed the former to study drag reduction. ${ }^{3}$ However, when the ball had to fall a long distance to reach a terminal velocity or when the falling velocity was large due to the use of a high density ball, the ball showed zig-zag motion, making measurement of its velocity very difficult.
Hence, we modified the latter and used it for the present study.

Drag reduction so far has been investigated mostly in aqueous solutions of nonionic polymers such as polyethylene oxide and polyacrylamide, and very little in aqueous polyelectrolytes. $\mathrm{Kim}$ and his coworkers ${ }^{4}$ examined the effect of the degree of neutralization on drag reduction due to aqueous poly(acrylic acid) and found that the percentage drag reduction increased with an increase in the degree of neutralization up to 0.46 and then leveled off at higher degrees of neutralization. This finding is of interest in relation to the theory of Kohn, ${ }^{5}$ who proposed that the origin of drag reduction due to a polymer solution is the deformation of polymer chains to an extended form by local stress in the turbulent sublayer.

In this paper, the principle of the rolling ball method is described in detail and the effect of added $\mathrm{KNO}_{3}$ on drag reduction due to aqueous sodium polyacrylate is discussed.

\section{EXPERIMENTAL}

\section{Materials}

A commercial sodium polyacrylate (NaPA) 
sample, manufactured by Kishida Chemical Company and designated as "high viscosity," was used. Its molecular weight was estimated to be $3.1 \times 10^{6}$ from a viscosity measurement. The potassium nitrate $\mathrm{KNO}_{3}$, used was of guaranteed reagent grade. These substances were used as delivered.

\section{Apparatus and Experimental Method}

Figure 1 illustrates schematically the rolling ball viscometer used. A steel ball of diameter $d$ rolls down in a glass tube of diameter $D$.

In order to cause turbulent flows in our apparatus it was necessary to allow the ball to fall at a high speed and thus the diameter ratio $d / D$ was chosen smaller than when the rolling ball method was used for the viscosity measurement. High velocities of the falling ball require a sufficiently long tube, since otherwise a terminal velocity can not be attained. The necessary tube length $L$ was estimated to be about one meter.

Two photogates were placed at 0.05 and $0.25 \mathrm{~m}$ from the lower end of the tube. A crystal oscillator stopwatch was used to measure the time in which the ball passes these photogates. The stopwatch was operated automatically by electrical signals from the photogates.

The velocity of the falling ball was measured at various inclination angles $\theta$ of the tube and the onset of drag reduction due to the polymer solution was detected when the ball velocity exceeded that in pure water.

The ball fell in a zig-zag fashion at $\theta$ near zero degree and slipped down, instead of rolling, on the tube wall at $\theta$ larger than 60 degrees. Therefore, the

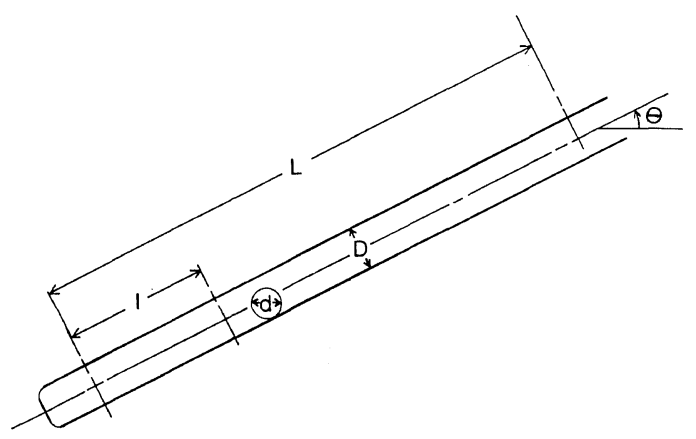

Figure 1. Schematic diagram of the apparatus used. $d$, diameter of the steel ball; $D$, diameter of the glass tube; $\theta$, inclination of the glass tube. experiment was limited to $\theta$ from 20 to 60 degrees.

The Reynolds number $R e$ is a function of fluid flow velocity, and this velocity was varied by changing $\theta$ in the present study.

The ball diameter was $9.51 \times 10^{-3} \mathrm{~m}$. The inner diameter of the tube was $1.55 \times 10^{-2} \mathrm{~m}$. Thus the diameter ratio $d / D$ was 0.614 .

The tube was surrounded by a water jacket in which thermostated water was circulated. The maximum temperature deviation was $0.10^{\circ} \mathrm{C}$ at $25^{\circ} \mathrm{C}$. The temperature for measurement was varied from 5 to $60^{\circ} \mathrm{C}$.

The viscosity of each sample liquid was measured by use of a Ubbelohde capillary viscometer.

\section{Calculation of the Average Flow Velocity and the Drag Coefficient}

This was carried out using the methods of Hubbard and Brown, ${ }^{6}$ which are outlined as follows.

The volume rate of the liquid pushed out by falling of the ball is $\pi d^{2} V / 4$, where $d$ is the diameter of the ball and $V$ the falling velocity of the ball. This rate is equal to the volume rate of the liquid passing through the channel between the ball and the tube wall. The latter is given by $\pi\left(D^{2}-d^{2}\right) \bar{v} / 4$, where $D$ is the inner diameter of the tube and $\bar{v}$ the average flow velocity in the channel considered. Thus, we obtain

$$
\bar{v}=\frac{d^{2}}{D^{2}-d^{2}} V
$$

According to Hubbard and Brown, ${ }^{6}$ the following relation can be derived by dimentional analysis:

$$
F /\left(h^{2} \rho \bar{v}^{2}\right)=\Phi(h \bar{v} \rho / \eta)
$$

where $F$ is the force driving the ball, $h$, the equivalent diameter of the tube, $\rho$, the density of the liquid, and $\eta$, the viscosity of the liquid. The quantity on the left-hand side of eq 2 is the drag coefficient $C_{\mathrm{D}}$. Thus

$$
C_{\mathrm{D}}=F /\left(h^{2} \rho \bar{v}^{2}\right)
$$

On the other hand, the variable for the function $\Phi$ in eq 2 is the Reynolds number $R e$. Thus

$$
R e=h \bar{v} \rho / \eta
$$

The force $F$ due to gravity is given by

$$
F=\frac{5}{7} g(\sin \theta) \frac{\pi d^{3}}{6}\left(\rho_{\mathrm{s}}-\rho\right)
$$




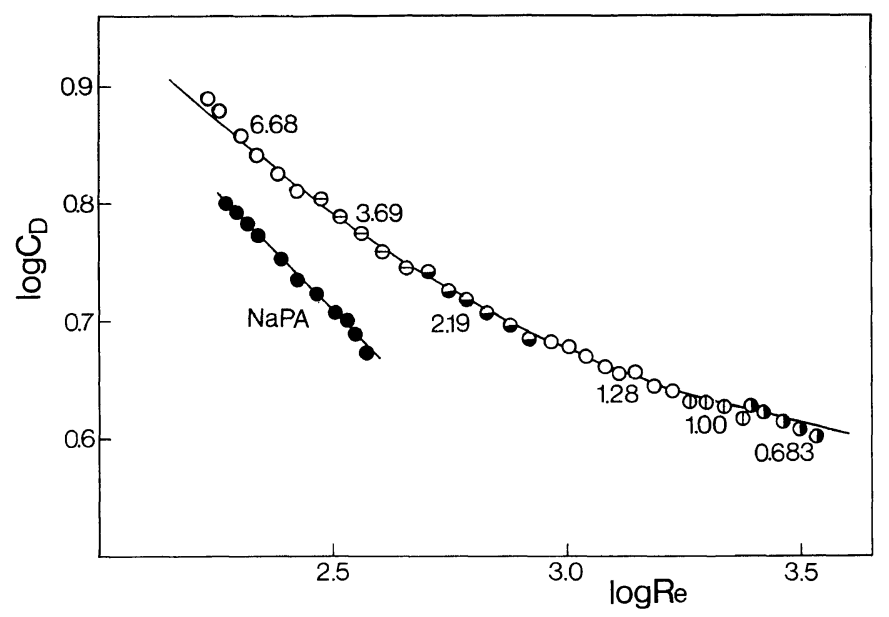

Figure 2. Double logarithmic plots of drag coefficient $C_{\mathrm{D}}$ against Reynolds number $R e$. The upper curve fits data for aqueous solutions (pure water and aqueous glycerol) containing no polymer; attached figures indicate the relative viscosities of the respective solutions at $25^{\circ} \mathrm{C}$.

Here, the coefficient $5 / 7$ is the fraction of the gravity $g$ effective for causing the translational motion of the rolling ball. The equivalent diameter $h$ is equal to four times the hydraulic diameter. Hence

$$
h=4 \times \frac{(\pi / 4)\left(D^{2}-d^{2}\right)}{\pi(D+d)}=D-d
$$

\section{RESULTS AND DISCUSSION}

The critical Reynolds number is defined as the $R e$ value at which the flow pattern changes from laminar to turbulent. It is well known that this value is about $10^{3}$ for pipe flow. Hubbard and Brown ${ }^{6}$ showed that the critical Reynolds number determined by the rolling ball method did not exceed $10^{2}$ and decreased with a decrease in $d / D$. Since $d / D$ of our apparatus was smaller than that of theirs, it is quite likely that our experiment was concerned with turbulent flow.

Hubbard and Brown ${ }^{6}$ also showed that when plotted double-logarithmically, the experimental data for $C_{\mathrm{D}}$ as a function of $R e$ followed a straight line of slope -1 in the laminar flow region and fell on a single concave-upward curve in the turbulent flow region. The upper group of data points in Figure 2 shows the values of $\log C_{\mathrm{D}}$ plotted against $\log R e$ for a series of aqueous solutions containing no polymer but different in relative viscosity. Experimentally, these different viscosities were ob-

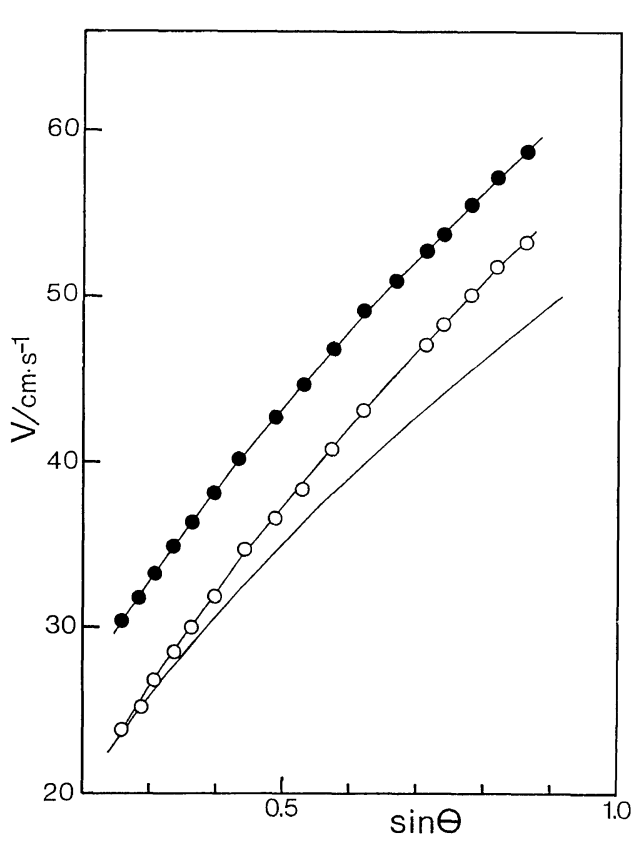

Figure 3. Ball velocity $V$ as a function of $\sin \theta$ for pure water (O) and aqueous NaPA (200 ppm) (O). The lower solid line is for the hypothetical liquid containing no polymer but having the same viscosity as the aqueous NaPA (200 ppm).

tained by changing the temperature of the solution or by adding glycerol to water. It can be seen that the plotted points fall on a single concave-upward 
curve, indicating that turbulent flows can be realized under our experimental conditions.

The polymer degradation often reported in studies using the pipe flow method was not found here. This was checked by measuring the viscosity of test solutions before and after drag reduction measurements.

The lower group of data points (filled circles) in Figure 2 shows $\log C_{\mathrm{D}}$ as a function of $\log R e$ for aqueous $\mathrm{NaPA}$ of $2.13 \times 10^{-3}$ monomol $1^{-1}$ $(200 \mathrm{ppm})$. These points appear below the reduced curve fitting the points for aqueous solutions containing no polymer, indicating that the addition of NaPA to water is effective for reducing the drag coefficient.

Figure 3 illustrates the $\theta$ dependence of the ball velocity $V$. It can be seen that $V$ for the NaPA solution is smaller than that for aqueous solutions containing no NaPA throughout the entire range of $\sin \theta$ studied, indicating that no drag reduction occurs by the addition of NaPA. This finding contradicts the data shown in Figure 2. However, this is only an apparent contradiction due simply to the higher viscosity and hence the lower Reynolds number of the NaPA solution. In other words, it is due to the fact that the two sets of data points in Figure 3 do not refer to the same Reynolds number. The following calculation makes this point clear.

Consider a hypothetical liquid containing no polymer but having the same viscosity as the $\mathrm{NaPA}$ solution studied. The $C_{\mathrm{D}} v s$. Re relation for this liquid should follow the upper reduced curve in Figure 2. Given a value for $R e, \bar{v}$ and $V$ can be computed from eq 1 and 4 . With this $\bar{v}$ and the $C_{\mathrm{D}}$ value interpolated at the given $R e$ from the upper reduced curve in Figure 2, $\sin \theta$ can be computed using eq 3 and 5. Similar calculations for a series of Re give $V$ as a function of $\sin \theta$. This function is shown as the lower solid curve in Figure 3. It can be seen that this curve appears below the line for the NaPA solution, indicating that when compared at the same viscosity, the addition of NaPA leads to an increase in $V$ regardless of $\theta$, i.e., a drag reduction.

In connection with the above consideration on the viscosity effect, it is interesting to examine what happens to drag reduction when the viscosity of the NaPA solution is diminished. As is well known, the viscosity of a polyelectrolyte solution decreases by the addition of a simple electrolyte. For NaPA, this was demonstrated by Takahashi et al. ${ }^{7}$ In the present study, $\mathrm{KNO}_{3}$ was chosen for the viscosity reduction.

Figure 4 shows $\log C_{\mathrm{D}} v s . \log$ Re plots for aqueous $\mathrm{NaPA}$ containing various amounts of $\mathrm{KNO}_{3}$. It can be seen that there occurs a rather sharp downward bending of the plot with the addition of the salt. This behavior indicates that the viscosity reduction due to the addition of $\mathrm{KNO}_{3}$ enhances drag re-

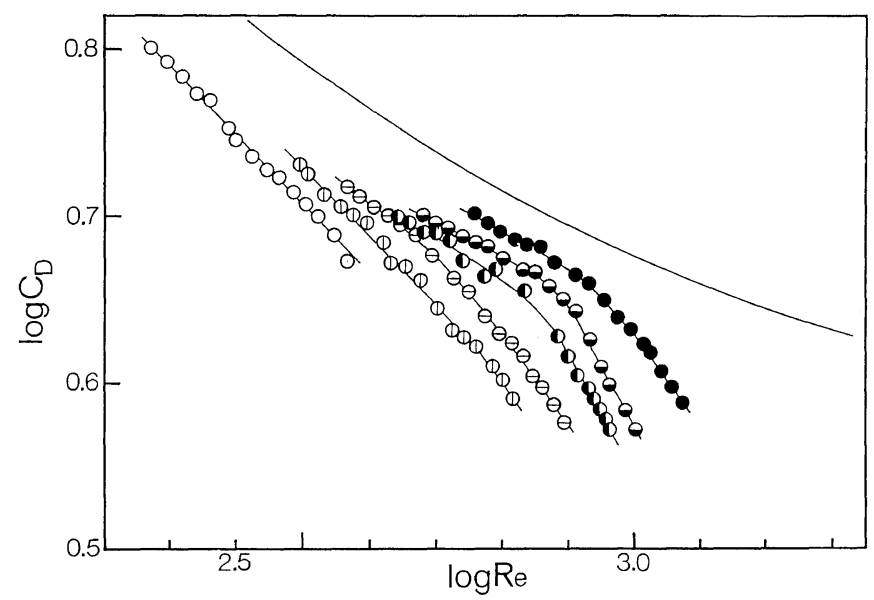

Figure 4. Double logarithmic plots of drag coefficient $C_{\mathrm{D}}$ against Reynolds number $R e$ for aqueous NaPA (200 ppm) containing various amounts of $\mathrm{KNO}_{3}$. $\bigcirc$, zero; (1), $1.00 \times 10^{-3} \mathrm{moll}^{-1} ; \Theta, 2.50 \times 10^{-3}$ moll $1^{-1} ; \circlearrowleft, 5.00 \times 10^{-3} \mathrm{moll}^{-1} ; \ominus, 7.50 \times 10^{-3} \mathrm{moll}^{-1} ; \bigcirc, 1.00 \times 10^{-2} \mathrm{moll}^{-1}$. The upper solid curve is a reproduction of the upper reduced curve in Figure 2. 
Drag Reduction

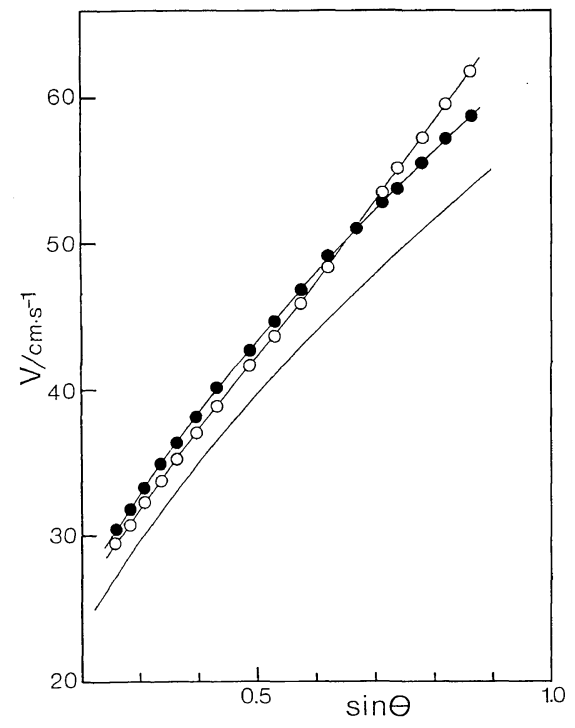

Figure 5. Ball velocity $V$ as a function of $\sin \theta$ for pure water (O) and aqueous NaPA (200 ppm) containing $5.00 \times 10^{-3} \mathrm{moll}^{-1} \mathrm{KNO}_{3}(\mathrm{O})$. The lower solid curve is for the hypothetical liquid containing no polymer but having the same viscosity as the aqueous NaPA (200 ppm) containing $5.00 \times 10^{-3} \mathrm{moll}^{-1} \mathrm{KNO}_{3}$.

duction. In Figure 5, the $V v s \cdot \sin \theta$ relation for the NaPA solution cotaining $5 \times 10^{-3} \mathrm{~mol}^{-1} \mathrm{KNO}_{3}$ is compared with that for pure water. The ball velocity in the former is seen to exceed that in the latter at a $\sin \theta$ value of about 0.7 . This finding is consistent with the results shown in Figure 4, that drag reduction is enhanced by the addition of $\mathrm{KNO}_{3}$.

In Figure 4, the data points counted at the same number from the ends of the respective curves correspond to the same experimental conditions except for the salt concentration. If these points are connected by a smooth curve, it is found that $C_{\mathrm{D}}$ of aqueous NaPA for fixed measuring conditions decreases with increasing $\mathrm{KNO}_{3}$ concentration up to $5 \times 10^{-3} \mathrm{~mol} \mathrm{l}^{-1}$ and increases with further addition of $\mathrm{KNO}_{3}$, in contrast to the viscosity of this polymer in water which decreases monotonically with increasing salt concentration. This finding was apparent from the $V v s$. $\sin \theta$ relation, and the maximum $V$ was found in the solution containing $5 \times 10^{-3} \mathrm{~mol} \mathrm{l}^{-1} \mathrm{KNO}_{3}$ (see Figure 5).

$\mathrm{Kohn}^{5}$ proposed an energy storage theory for the drag reduction due to polymer solutions. When the critical Reynolds number is exceeded, the kinetic energy is dissipated as a result of turbulent mixing of fluid particles. However, when polymer molecules are present in the fluid, they respond to local stress by a conformation change and absorb and store energy in a deformed state. Thus, the polymer chains act as an energy sink and should lead to a drag reduction.

According to this theory, our finding concerning the dependence of $C_{\mathrm{D}}$ on salt concentration may be interpreted as follows. When the $\mathrm{KNO}_{3}$ concentration is low, the polymer chain is so expanded due to strong electrostatic repulsions that it is less easily deformable and less effective for absorbing the kinetic energy of the fluid. Thus, at low $\mathrm{KNO}_{3}$ concentrations, no appreciable drag reduction takes place. At high $\mathrm{KNO}_{3}$ concentrations, on the other hand, the polymer chain is highly coiled and has smaller dimensions as a result of the screening of electrostatic repulsions by the added salt. Such polymer chains may not effectively absorb energy from the fluid and thus may not cause marked drag reduction. At intermediate $\mathrm{KNO}_{3}$ concentrations, the polymer chain should be relatively extended but still possess some flexibility, thus being able to effect a maximum energy absorption. In this way, it may be understood why we observed a maximum drag reduction at a $\mathrm{KNO}_{3}$ concentration of $5 \times 10^{-3} \mathrm{~mol}$ $1^{-1}$ when $\mathrm{KNO}_{3}$ added to an aqueous $\mathrm{NaPA}$ was varied from zero to $1 \times 10^{-2} \mathrm{~mol}^{-1}$.

\section{REFERENCES}

1. B. A. Toms, Proc. 1st Intern. Congr. Rheol., Vol. II, North Holland, Amsterdam, 1949, p 135.

2. F. Yamashita, K. Nakamura, and T. Nakagawa, Rep. Prog. Polym. Phys. Jpn., 24, 93 (1981).

3. K. Imahara, the Master's Thesis, Hokkaido University, 1981.

4. O. K. Kim, R. C. Rittle, and R. Y. Ting, AIChE., Symp., 69, 39 (1973).

5. M. C. Kohn, J. Polym. Sci., Polym. Phys. Ed., 11, 2339 (1973).

6. R. M. Hubbard and G. G. Brown, Ind. Eng. Chem., 15, 212 (1943).

7. A. Takahashi, S. Kamei, and I. Kagawa, J. Chem. Soc. Jpn., Chem. Ind. Chem., 83, 14 (1962). 